\title{
Sports Genomics and Sport Doping
}

\author{
By Bogdan-Alexandru Hagiu ${ }^{*}$ \& Cristina-Mihaela Ghiciuc ${ }^{ \pm}$
}

\begin{abstract}
A topical issue is the need to identify new possibilities for doping, in order to protect the athlete's privacy. We consider it useful to explore the relationship between gene variants that predispose to sports performance and special sensitivity to certain doping substances. For this purpose, we conducted a study in the literature. Among the substances banned from athletes, there are some whose effect is amplified in carriers of gene variants that confer special sports skills anyway: meldomium (athletes with overexpression of PEPCK-C), corticotrophins and their releasing factors (carriers of the allele that produces alpha-actinin-3), caffeine (athletes careers of PPARA genotypes intron 7 rs4253778 CC and Leu162Val rs1800206 CG), somatotrope (polymorphism rs8192678 of PPARGC1A, polymorphism rs11549465 C>T of HIF1A gene), beta-blockers (carriers of the ACE DD genotype), insulin and its mimetics (rs11549465 C>T polymorphism of the HIF1A gene), cannabinoids ( $\alpha, \beta$ and $\gamma$ isoforms of PPAR), stimulants and narcotics (carriers of RBFOX1 rs7191721 $G$ ), anabolic steroids (athletes carrying EPOR that provide an advantage for physical effort). A number of drugs, supplements, hormones, flavonoids, vitamins and experimental therapeutic substances that are not on the WADA 2020 list may be the subject of future studies regarding their potential as doping substances, given that they have a stimulating action on exercise capacity in the case of particular genomes: cortisol blockers (carriers of the allele that produces alpha-actinin-3), carbohydrate and lipid supplements (polymorphism rs8192678 of PPARGC1A), quercetin (genotypes PPARA intron 7 rs4253778 CC and și PPARA Leu162Val rs1800206 CG), AMPD inhibitors (AMPD1 Gln12 carriers), folates (carriers of the MTHFR polymorphism rs1801131 C), nitrates (variants of NOS3 rs2070744 T), saroglizatar and lobeglitazone (carriers of the Pro12Ala polymorphism), thyroid hormones (variant EP441s, variant TSHR C), Tribulus terrestris (AR variant).
\end{abstract}

Keywords: Gene variants, potential doping

\section{Introduction}

In the literature it has been discussed that analysis and the developed guiding reference are necessary to highlight the need for scientists/clinicians to be wellversed in ethics and data protection policy to advance sport and exercise genomics without compromising the privacy of athletes and the efforts of international sports federations (Tanisawa et al. 2020). However, it can be considered that in order to establish some rules, in addition to the correlations between nutrition and genomics in sports (Guest et al. 2019) and the possible links between gene variants

\footnotetext{
*Associate Professor, Faculty of Physical Education and Sport, "Alexandru Ioan Cuza" University, Romania.

${ }^{ \pm}$Professor, Faculty of Medicine, "Gr. T. Popa" University of Medicine and Pharmacy, Romania.
} 
that favor sports talent and doping possibilities must be known. There are functional proteins with potential for genetic doping (erythropoietin, insulin-like growth factor, growth hormone, myostatin, vascular endothelial growth factor, fibroblast growth factor, endorphin and enkephalin, $\alpha$ actinin 3, peroxisome proliferator-activated receptor-delta (PPAR $\delta$ ) and cytosolic phosphoenolpyruvate carboxykinase (PEPCK-C) but also genes with high potential for abuse (PPAR $\delta$ and PEPCK-C) (van der Gronde et al. 2013). There were identified 11 genetic markers that create skills for endurance (ACE I, ACTN3 577X, PPARA rs4253778 G, PPARGC1A Gly482) and for strength exercises (ACE D, ACTN3 Arg577, AMPD1 Gln12, HIF1A 582Ser, MTHFR C, rs2070744 T, PPARG 12Ala), and also an number of markers that have been highlighted in athletes of different nationalities, belonging to different sports (CREM rs1531550 A, DMD rs939787 T, GALNT13 rs10196189 G, NFIA-AS1 rs1572312 G, R and TSHR rs7144481 C) (Ahmetov and Fedotovskaya 2015). There have been situations when a single gene variant leads to increased athletic performance, such as erythropoietin receptor (EPOR) or androgen receptor (AR), or when the athletic advantage is the prerogative of hormonal excesses or deficiencies (virilizing adrenal hyperplasia, deficiency genetics of growth hormone, myostatin deficiency) or even genetic diseases (partial familial lipodystrophy, which affects the female gender, some varieties being produced by LMNA gene damage) (Rogol and Pieper 2017). Considering these aspects, the paper aims, based on data from the literature, to identify substances that can act on gene variants that increase sports performance, so as to amplify their effects. Once these substances were identified, we set out to establish, based on the critical analysis of the literature, which of them are on the WADA 2020 list and which can be subsequently introduced on the list of substances banned from athletes carrying particular gene variants.

\section{Method}

A literature search was conducted to identify doping substances or those with doping potential to which athletes wearing gene variants that facilitate performance are more sensitive, using articles especially from PubMed, published between 2001 and 2020. In principle, the keywords athletic, doping, drugs, and genes were used, but the publications derived from the relevant identified works were also searched, following the questions "Which gene variants influence sports performance?" and "What changes in drug metabolism induce those genes?"

\section{Review of the Literature}

Cytosolic phosphoenolpyruvate carboxykinase (PEPCK-C) does not singularly control the rate of hepatic gloconeogenesis, and an activation of the tricarboxylic acid cycle is also required (Burgess et al. 2007).

The ACTN3 gene encodes the $\alpha$-actinin-3 protein, which stabilizes the contractile apparatus at the Z-lines, and the ACTN3 R577X polymorphism could 
be a new target for readthrough therapy that may affect human athletic and muscular performance (Harada et al. 2018).

In footballers, the presence of the allele that produces alpha-actinin-3 shows higher values of testosterone after the game compared to the control group, as well as cortisol, a fact related to the increased values of markers of muscle microtrauma and hormonal stress, both probably produced by more speed and power actions during the game (Coelho et al. 2019).

Cortisol has not been shown to be effective in increasing performance, but athletes have an anabolic/catabolism balance at lower values than untrained individuals, as indicated by the testosterone/cortisol ratio (Dvorak et al. 2006).

The effects of sports training on the genotype PPARA intron $7 \mathrm{rs} 4253778 \mathrm{CC}$ are increased concentrations of low-density lipoproteins and blood glucose, and on the genotype PPARA Leu162Val rs1800206 CG - decrease in the concentration of high-density lipoproteins (HDL) (Maciejewska-Skrendo et al. 2019). However, the results of quercetin supplementation in these careers may be different from those recorded in endurance runners, in which quercetin reduced lipid peroxidation but did not improve performance (Scholten and Sergeev 2013).

Also, career athletes of the PPARA intron 7 rs4253778 CC and Leu162Val rs1800206 CG genotypes could metabolize caffeine differently, a substance with action on lipid metabolism, banned by WADA (Macchiarella et al. 2017).

Regarding the peculiarities of carbohydrate metabolism, the influences of growth hormone and insulin on sports performance should not be neglected (Sonksen 2001).

Even if the effect is minimal, the rs 8192678 polymorphism of peroxisome proliferator-activated receptor- $\gamma$ co-activator 1- $\alpha$ (PPARGC1A) may contribute to the development of metabolic syndrome (Csép et al. 2017), so carriers may have differences in the metabolism of quercetin, carbohydrate and lipidic supplements, caffeine, insulin and somatotropes.

Angiotensin (ACE) converting enzyme allele ACE-D in patients with heart failure has an increased ACE activity, and the effects of beta-blockers and high doses of ACE inhibitors have been shown to be more pronounced in those with the ACE DD genotype (McNamara et al. 2004). If the results can be extrapolated to healthy individuals, given that beta-blockers can reduce tremor in some sports, such as shooting and golf (Hughes 2015), it turns out that ACE DD carriers may benefit from using beta-blockers as doping substances.

The role of AMPD1 Gln12 in increasing sports performance can be explained by the fact that pharmacological inhibition of AMPD potentiates energy-producing cellular enzymes, without influencing glucose transport (Plaideau et al. 2014).

The rs11549465 C>T polymorphism of the HIF1A gene facilitates glucose metabolism (Gabbasov et al. 2013), which means that carriers of this variant would require lower doses of caffeine, insulin or somatotrope to increase athletic performance.

The MTHFR gene (methylenetetrahydrofolate reductase) is crucial in folate metabolism (Kao et al. 2014), which may mean a more efficient use of folic acid supplements in carriers of the MTHFR rs1801131 C polymorphism.

There are variants of NOS3 rs2070744 T which, possibly due to influences on 
striated muscle metabolism, affect nitrite/nitrate levels in patients with idiopathic intolerance to environmental factors (De Luca et al. 2015).

For CrossFit-trained athletes, the effects of six days of nitrate supplementation are reduced metabolic cost of exercise, increased peak strength and increased maximum strength (Kramer et al. 2016). Nitrate supplementation may also bring some benefits in antioxidant defense and blood pressure regulation after aerobic exercise (Menezes et al. 2019).

Regarding the association of exercise skills and Pro12Ala polymorphism of the gamma receptor gene of peroxisome proliferation activation, 12Ala allele carriers have increased insulin sensitivity, leading to more efficient use of glucose in active skeletal muscle (Maciejewska-Karlowska et al. 2013).

PPAR $\delta$ not only has high potential for abuse, but retains glucose reserves, being worthy of consideration for the potential of PPAR $\delta$-targeted exercise mimetics in the enhancement of athletic performance (Fan et al. 2017).

The functions of the PPAR gene open up new potential perspectives for doping in sport. Thus, fibrates (PPAR- $\alpha$ agonists) and thiazolidinediones (PPAR- $\gamma$ agonists) are used extensively in clinical practice to correct dyslipidemia and lower blood glucose, but two PPAR- $\alpha / \gamma$ agonists, saroglitazar and lobeglitazone, have also been manufactured (Han et al. 2017).

Some isoforms of PPAR ( $\alpha, \beta$ and $\gamma$ ) can be activated by cannabinoids (O'Sullivan 2016). Cannabis has a potential impact on athletes' health, but also on performance, both during training and competition. The potential beneficial effects of cannabis as part of a pain-relieving protocol, including reducing emotionrelated symptoms, deserve further attention (Ware et al. 2018).

The RBFOX1 gene (RBFOX1 rs7191721 G being a genetic marker identified in elite athletes (Ahmetov and Fedotovskaya 2015), is a coordinating factor of transcriptional programs necessary for neuronal development (Fogel et al. 2012), so it can be assumed that RBFOX1 rs7191721 G carriers require lower doses of doping substances with neuronal tropism (stimulants, narcotics, cannabinoids, banned according to WADA 2020), to increase athletic performance.

The TSHR gene causes the synthesis of receptors that attach to thyroid stimulating hormone (TSH), so it is possible that the reactivity of individuals with TSHR variant rs7144481 C (which increases sports skills) to thyroid hormones is higher, resulting in improvement performance (Ahmetov and Fedotovskaya 2015).

Erythropoietin and agents that affect erythropoiesis are banned by WADA 2020 , and this can be particularly noticeable for carriers of the genetic variant with erythropoietin receptors (EPOR) that provide athletic advantage, and for those with androgenic receptor (AR) variants that amplify the action. Hormone would theoretically require lower doses of steroid anabolic agents.

Experimentally, in rats, EPOR has been shown to correlate with chronic postlesional pain, with the respective receptors being expressed along the spinal axis in increased amounts after musculoskeletal trauma (Cohrs 2018).

Moreover, a study conducted on breast cancer cell lines revealed that membrane-acting androgens modify the transcription of the erythropoietin receptor (EPOR), leading to erythropoietin-initiated actions (Pelekanou 2010).

Experimentally, in rats that performed high-intensity exercise, it was found 
that treatment with Tribulus terrestris extract promotes increased muscle mass and performance by increasing testosterone and IGF 1 plasma levels, but also receptors for those hormones in the striated muscles (Wu et al. 2017).

\section{Discussion}

Athletes with overexpression of PEPCK-C also have an increased requirement for Krebs cycle constituents to sustain effort, from this point of view it is important to mention meldonium (a substance banned by WADA including in previous years), which reduces endogenous synthesis and biological activity of carnitine (Lippi 2017).

Cortisol blockers could be used in carriers of the allele that produces alphaactinin-3 to act with increased efficiency in order to increase muscle mass. Cortisol blockers are not on the list of substances banned by WADA 2020, instead corticotrophins and their releasing factors, yes.

Quercetin (which may favor PPARA intron 7 rs4253778 CC and PPARA Leu162Val rs1800206 CG genotypes for sports performance) is not included in the WADA 2020 list of prohibited substances. Caffeine (which may influence the sports performance of PPARA intron 7 rs4253778 CC and Leu162206 CG rP genotypes) is included in the 2020 Monitoring Program, not being considered a prohibited substance. Somatotrope, a hormone that can influence the adaptation to effort of the genotypes mentioned above, is on the list of banned substances WADA 2020.

Carbohydrate and lipid supplements, which could elicit a particular response to exercise adaptation in individuals with the rs8192678 polymorphism of peroxisome proliferator-activated receptor- $\gamma$ co-activator 1- $\alpha$ (PPARGC1A), are not on the WADA 2020 list.

WADA 2020 indicates beta-blockers as being banned intracompetitionally, but also out of competition for some sports. It should not be forgotten that ACE DD carriers may benefit from the use of beta-blockers as doping agents.

The data presented show that substances with the pharmacological property of inhibiting AMPD can increase athletic performance. A new generation of AMPD inhibitors is being tested (Zabielska et al. 2015) which may be potential doping substances in sports.

Insulin and its mimetics are on the list of prohibited substances (WADA 2020), and the rs $11549465 \mathrm{C}>\mathrm{T}$ polymorphism of the HIF1A gene requires lower doses of insulin to increase athletic performance, as in the case of caffeine and somatrotrope. Also, lower doses of insulin than those used for doping non-career individuals may, in carriers of the 12Ala allele, favor athletic performance. Folates are not on the WADA 2020 list, but folic acid supplements can be used more effectively in carriers of the MTHFR rs1801131 C polymorphism.

If data on NOS3 rs2070744 T variants affecting nitrite / nitrate levels in patients with idiopathic intolerance to environmental factors can be extrapolated to healthy subjects for whom the gene enhances athletic performance, those gene variants may also influence the effects of nitrate supplementation (their 
potentiation). Nitrates are not on the WADA 2020 list of banned substances, but could exert amplified effects on increasing athletic performance in those variants of NOS3 rs2070744 T.

PPAR agonists (saroglitazar and lobeglitazone) could be used to increase athletic performance due to the induction of hyperglycaemia, especially in carriers of the Pro12Ala polymorphism. Saroglizatar and lobeglitazone are not on the WADA 2020 list, but have doping potential for carriers of particular PPAR variants.

Cannabinoids, with the exception of cannabidiol, are banned by WADA 2020. Cannabinoids may potentiate the hyperglycemic effects of PPAR activation by specific agonists, saroglitazar and lobeglitazone, and the doping action is amplified. RBFOX1 rs7191721 G carriers may require lower doses of doping substances with neuronal tropism (stimulants, narcotics, cannabinoids, banned under WADA 2020) to increase athletic performance.

Although thyroid hormones are not found on the WADA 2020 list, they play fundamental roles in muscle physiology (Bloise et al. 2018) and may favor TSHR rs7144481 C carriers for achieving athletic performance.

It is possible that the careers of the EPOR variant that offers an athletic advantage may require lower doses of analgesic substances. The 2017 WADA prohibited list specifies that narcotics and cannabinoids are banned in competitions, but not other analgesics (Vernec et al. 2017), a fact maintained on the WADA 2020 list.

It would be possible to extrapolate that athletes, with EPOR that provide an advantage for physical effort, show not only better oxygenation of active skeletal muscle, but also an increased sensitivity to some anabolic steroids. Anabolic agents are banned under WADA 2020.

If Tribulus terrestris multiplied androgen receptors with increased potential for amplifying messenger proteins, as is the case with carriers of gene variants of AR that promote athletic performance, the effectiveness of this supplement to increase athletic performance would be much higher, perhaps gaining the value of a doping substance (Tribulus terrestris is not on the WADA 2020 list).

Of course, in order to evaluate the importance of these correlations on the reevaluation of the list of banned substances in sports, future studies of pharmacokinetics and even pharmacodynamics are needed, in correlation with the further identification of new genotypes that favor sports talent. It is thus necessary to study in particular the time of elimination of prohibited substances or with prospects to be added to the list of prohibited substances in the respective subjects, as well as the possibilities of identification (sensitive tests). Another direction is the identification of gene variants that intervene in the metabolism of doping or potentially doping substances and the investigation of the effect of their coexistence with genomes that favor sports talent.

For example, for testosterone, variants ESR2 (rs928554) and SHBG (rs1799941) result in a smaller number of copies of AR (Celec et al. 2013). For cannabinoids, H316Y and Q63R / H316Y polymorphic receptors exhibited higher constitutive activity than the CB2 wild-type receptor (Carrasquer et al. 2010). In addition to PPAR $\gamma$, there are many gene variants that alter insulin resistance, 
including IRS1, COBLL1-GRB14, PPP1R3B, PDGFC, UHRF1BP1 and LYPLAL1 (Brown and Walker 2016). Genetic variants that alter the associations between dietary factors and sports performance have been highlighted for caffeine (CYP1A2 (rs762551), ADORA2A (rs5751876)) and folate (MTHFR (rs1801133) (Guest et al. 2019).

\section{Conclusions}

The analysis of data from the literature suggests the existence of two categories of substances that have the potential to increase sports performance in individuals who have a genome that promotes sports talent:

- Drugs and hormones banned in sports, with an amplified action on some gene variants: meldonium, corticotrophins and their releasing factors, caffeine, somatotrope, beta-blockers, insulin and its mimetics, cannabinoids, stimulants, narcotics, anabolic steroids.

- Drugs, supplements, hormones, flavonoids, vitamins and experimental therapeutic substances that are not on the WADA 2020 list, but which may be the subject of future studies regarding their potential as doping substances: cortisol blockers, carbohydrate supplements, lipid supplements, quercetin, AMPD inhibitors, folates, nitrates, saroglizatar, lobeglitazone, thyroid hormones, analgesics, Tribulus terrestris.

\section{References}

Ahmetov II, Fedotovskaya ON (2015) Current progress in sports genomics. Advances in Clinical Chemistry 70(Aug): 247-314.

Bloise FF, Cordeiro A, Ortiga-Carvalho TM (2018) Role of thyroid hormone in skeletal muscle physiology. The Journal of Endocrinology 236(1): R57-R68.

Brown AE, Walker M (2016) Genetics of insulin resistance and the metabolic syndrome. Current Cardiology Reports 18(8): 1-8.

Burgess SC, He T, Yan Z, Lindner J, Sherry AD, Malloy CR et al. (2007) Cytosolic phosphoenolpyruvate carboxykinase does not solely control the rate of hepatic gluconeogenesis in the intact mouse liver. Cell Metabolism 5(4): 313-320.

Carrasquer A, Nebane NM, Williams WM, Song ZH (2010) Functional consequences of nonsynonymous single nucleotide polymorphisms in the CB2 cannabinoid receptor. Pharmacogenetics and Genomics 20(3): 157-166.

Celec P, Tretinárová D, Minárik G, Ficek A, Szemes T, Lakatošová S et al. (2013) Genetic polymorphisms related to testosterone metabolism in intellectually gifted boys. PloS One 8(1): e54751.

Coelho DB, Pimenta EM, Rosse IC, Veneroso C, Pussieldi GA, Becker LK et al. (2019) Alpha-actinin-3 R577X polymorphism influences muscle damage and hormonal responses after a soccer game. Journal of Strength and Conditioning Research 33(10): 2655-2664.

Cohrs G, Goerden S, Lucius R, Synowitz M, Mehdorn HM, Held-Feindt J et al. (2018) Spatial and cellular expression patterns of erythropoietin-receptor and erythropoietin 
during a 42-day post-lesional time course after graded thoracic spinal cord impact lesions in the rat. Journal of Neurotrauma 35(3): 593-607.

Csép K, Szigeti E, Vitai M, Korányi L (2017) The PPARGC1A - GLY482SER polymorphism (RS8192678) and the metabolic syndrome in a central romanian population. Acta Endocrinologica 13(2): 161-167.

De Luca C, Gugliandolo A, Calabrò C, Currò M, Ientile R, Raskovic D et al. (2015) Role of polymorphisms of inducible nitric oxide synthase and endothelial nitric oxide synthase in idiopathic environmental intolerances. Mediators of Inflammation 7(Mar): 245308.

Dvorak J, Feddermann N, Grimm K (2006) Glucocorticosteroids in football: use and misuse. British Journal of Sports Medicine Suppl 1 40(Jul): i48-i54.

Fan W, Waizenegger W, Lin CS, Sorrentino V, He MX, Wall CE et al. (2017) PPAR $\delta$ promotes running endurance by preserving glucose. Cell Metabolism 25(5): 11861193.

Fogel BL, Wexler E, Wahnich A, Friedrich T, Vijayendran C, Gao F et al. (2012) RBFOX1 regulates both splicing and transcriptional networks in human neuronal development. Human Molecular Genetics 21(19): 4171-4186.

Gabbasov RT, Arkhipova AA, Borisova AV, Hakimullina AM, Kuznetsova AV, Williams AG et al. (2013) The HIF1A gene Pro582Ser polymorphism in Russian strength athletes. Journal of Strength and Conditioning Research 27(8): 2055-2058.

Guest NS, Horne J, Vanderhout SM, El-Sohemy A (2019) Sport nutrigenomics: personalized nutrition for athletic performance. Frontiers in Nutrition 6(8): 1-16.

Han L, Shen WJ, Bittner S, Kraemer FB, Azhar S (2017) PPARs: regulators of metabolism and as therapeutic targets in cardiovascular disease. Part II: PPAR- $\beta / \delta$ and PPAR- $\gamma$. Future Cardiology 13(3): 279-296.

Harada N, Hatakeyama A, Okuyama M, Miyatake Y, Nakagaw T, Kuroda M et al. (2018) Readthrough of ACTN3 577X nonsense mutation produces full-length $\alpha$-actinin-3 protein. Biochemical and Biophysical Research Communications 502(3): 422-428.

Hughes D (2015) The world anti-doping code in sport: update for 2015. Australian Prescriber 38(5): 167-170.

Kao AC, Rojnic Kuzman M, Tiwari AK, Zivković M, Chowdhury NI, Medved V et al. (2014) Methylenetetrahydrofolate reductase gene variants and antipsychotic-induced weight gain and metabolic disturbances. Journal of Psychiatric Research 54(1): 3642.

Kramer SJ, Baur DA, Spicer MT, Vukovich MD, Ormsbee MJ (2016) The effect of six days of dietary nitrate supplementation on performance in trained CrossFit athletes. Journal of the International Society of Sports Nutrition 13(1): 1-7.

Lippi G, Mattiuzzi C (2017) Misuse of the metabolic modulator meldonium in sports. Journal of Sport and Health Science 6(1): 49-51.

Macchiarella A, Amato A, Sacco A, Rabboni M, Contrò V, Proia P (2017) Nutritional supplement habits: the survey on a Sicilian group. Trends in Sport Sciences 3(24): $123-127$.

Maciejewska-Karlowska A, Sawczuk M, Cieszczyk P, Zarebska A, Sawczyn S (2013) Association between the Pro12Ala polymorphism of the peroxisome proliferatoractivated receptor gamma gene and strength athlete status. PloS One 8(6): e67172.

Maciejewska-Skrendo A, Buryta M, Czarny W, Król P, Stastny P, Petr M et al. (2019) The Polymorphisms of the Peroxisome-Proliferator Activated Receptors' Alfa Gene Modify the Aerobic Training Induced Changes of Cholesterol and Glucose. Journal of Clinical Medicine 8(7): 1043.

Menezes EF, Peixoto LG, Teixeira RR, Justino AB, Puga GM, Espindola FS (2019) Potential benefits of nitrate supplementation on antioxidant defense system and blood 
pressure responses after exercise performance. Oxidative Medicine and Cellular Longevity (Mar): 1-10, 7218936.

McNamara DM, Holubkov R, Postava L, Janosko K, MacGowan GA, Mathier M, Murali et al. (2004) Pharmacogenetic interactions between angiotensin-converting enzyme inhibitor therapy and the angiotensin-converting enzyme deletion polymorphism in patients with congestive heart failure. Journal of the American College of Cardiology 44(10): 2019-2026.

O'Sullivan SE (2016) An update on PPAR activation by cannabinoids. British Journal of Pharmacology 173(12): 1899-1910.

Pelekanou V, Notas G, Sanidas E, Tsapis A, Castanas E, Kampa M (2010) Testosterone membrane-initiated action in breast cancer cells: Interaction with the androgen signaling pathway and EPOR. Molecular Oncology 4(2): 135-149.

Plaideau C, Lai YC, Kviklyte S, Zanou N, Lofgren L, Andersen H, et al. (2014) Effects of pharmacological AMP deaminase inhibition and Ampd1 deletion on nucleotide levels and AMPK activation in contracting skeletal muscle. Chemistry \& Biology 21(11): 1497-1510.

Rogol AD, Pieper LP (2017) Genes, gender, hormones, and doping in sport: a convoluted tale. Frontiers in Endocrinology 8(Oct): 251.

Scholten SD, Sergeev IN (2013) Long-term quercetin supplementation reduces lipid peroxidation but does not improve performance in endurance runners. Open Access Journal of Sports Medicine 4(Mar): 53-61.

Sonksen PH (2001) Insulin, growth hormone and sport. The Journal of Endocrinology 170(1): 13-25.

Tanisawa K, Wang G, Seto J, Verdouka I, Twycross-Lewis R, Karanikolou A et al. (2020) Sport and exercise genomics: the FIMS 2019 consensus statement update. British Journal of Sports Medicine (Mar): bjsports-2019-101532.

van der Gronde T, de Hon O, Haisma HJ, Pieters T (2013) Gene doping: an overview and current implications for athletes. British Journal of Sports Medicine 47(11): 670-678.

Vernec A, Pipe A, Slack A (2017) A painful dilemma? Analgesic use in sport and the role of anti-doping. British Journal of Sports Medicine 51(17): 1243-1244.

Ware MA, Jensen D, Barrette A, Vernec A, Derman W (2018) Cannabis and the health and performance of the elite athlete. Clinical Journal of Sport Medicine: Official journal of the Canadian Academy of Sport Medicine 28(5): 480-484.

World Anti-Doping Agency - WADA (2020) The world anti-doping code international standard: prohibited list. Retrieved from: https://www.wada-ama.org/sites/default/fi les/wada_2020_english_prohibited_list_0.pdf. [Accessed July 2020].

Wu Y, Yang H, Wang X (2017) The function of androgen/androgen receptor and insulin growth factor-1/insulin growth factor-1 receptor on the effects of Tribulus terrestris extracts in rats undergoing high intensity exercise. Molecular Medicine Reports 16(3): 2931-2938.

Zabielska MA, Borkowski T, Slominska EM, Smolenski RT (2015) Inhibition of AMP deaminase as therapeutic target in cardiovascular pathology. Pharmacological Reports: PR 67(4): 682-688. 
